\title{
Educação alimentar e nutricional da teoria à prática: um relato de experiência
}

\author{
Food and nutritional education from theory to practice: an experience report
}

Natália Luiza Kops ${ }^{1}$, Júlia Zys ${ }^{1}$, Maurem Ramos ${ }^{2}$

\begin{abstract}
RESUMO
Objetivo: Com o objetivo de desenvolver experiência na área de educação alimentar e nutricional, acadêmicas do curso de Nutrição realizaram ações educativas com alunos de uma escola pública de Porto Alegre. Buscou-se contribuir no conhecimento dos alimentos e na mudança de hábitos e práticas alimentares saudáveis dos escolares.

Descrição da experiência: Para tanto, durante a disciplina de Educação Nutricional, duas acadêmicas foram orientadas a realizar intervenções educativas lúdicas semanais com 17 escolares de 10 a 12 anos em um período de 2 meses. O primeiro passo foi o diagnóstico, em que se identificou um alto percentual de excesso de peso, além de alto consumo de frituras, guloseimas e refrigerantes. A partir desse dado, as ações foram planejadas.

Conclusão: Ficou evidente a importância de serem utilizados materiais ilustrativos para as ações, bem como vídeos breves e atrativos e abordagem dirigida ao perfil do público alvo. Através das intervenções realizadas, uma base de conhecimento foi construída, proporcionando aos escolares condições para que possam refletir e tomar decisões mediante fatos percebidos. A experiência adquirida prepara os graduandos a solucionarem imprevistos e a lidarem com ações educativas de acordo com as necessidades do público alvo.
\end{abstract}

Palavras-chave: educação alimentar e nutricional; consumo de alimentos; nutrição do adolescente.

\begin{abstract}
Objective: The present work aimed to develop an experience in the food and nutrition education area and, for that, academic students of Nutrition performed educational activities with students from a state school in Porto Alegre. Indeed, we aimed to contribute to a better food knowledge and to change food consumption healthy habits of the students.

Description of experience: In order to do so, during the Nutrition Education subject, two academic students were oriented to perform weekly-illustrated educational interventions with 17 students between 10 and 12 years old for two months. The first step was the diagnosis, where it was identified a high percentage of overweight, besides a high consumption of fried foods, sweets and soft drinks. Based on these data, educational actions were planned.

Conclusion: It became evident the importance of using playful materials in the activities, as well as brief and attractive videos and an approach that is adequate to the profile of the target public. Through the interventions performed, a knowledge basis was built, providing students with conditions to reflect and make decisions on perceived facts. The acquired experience prepares the graduating students to solve unexpected situations and to deal with educational actions according to the needs of the target group.
\end{abstract}

Keywords: food and nutrition education; food consumption; adolescent nutrition.

\footnotetext{
${ }^{1}$ Acadêmicas do Curso de Graduação em Nutrição, Universidade Federal do Rio Grande do Sul (UFRGS).

${ }^{2}$ Nutricionista. Doutorado em Ciências da Saúde pela UFRGS. Professora adjunto do Departamento de Medicina Social da UFRGS.
} 


\section{INTRODUÇÃo}

Experiências precoces e interação contínua com o alimento influenciam nas preferências alimentares, hábitos e atitudes exibidas pelos adultos ${ }^{1-6}$. Sabe-se que, quanto mais precoce for adotado um estilo de vida saudável, menor será o risco de doenças crônicas não transmissíveis no futuro. O ambiente escolar, por abordar um público jovem e por ser um local de ensino para formação de comportamentos saudáveis é um excelente local para se começar uma educação alimentar e nutricional ${ }^{5}$.

Pessoas com maior poder aquisitivo consideram a qualidade nutricional como um determinante na compra de alimentos ${ }^{7}$. O mesmo não acontece para indivíduos com menor escolaridade e nível socioeconômico mais baixo devido a influencia do preço e do acesso ao alimento $^{7}$. No entanto, mesmo com custo mais elevado, a facilidade e praticidade proporcionada por alimentos industrializados acabam, muitas vezes, se sobrepondo ao consumo de alimentos mais saudáveis ${ }^{8}$. O que pode explicar por que, nos últimos 34 anos, o índice de sobrepeso no Brasil aumentou seis vezes para meninos e três para meninas entre 10 e 19 anos $^{8}$.

Para mudanças destes dados epidemiológicos é preciso ampliar a disseminação de informações, aumentar a consciência e motivação do público alvo, bem como definir plano de ações educativas para promoção da saúde ${ }^{1}$. A educação alimentar e nutricional é uma ferramenta para contribuir com tais mudanças ${ }^{9}$. Uma abordagem educativa convencional, fundamentada apenas na transmissão de informações é, em geral, insuficiente para motivar mudanças mais significativas das práticas de saúde. Fatos relativos à ação fisiológica dos nutrientes e manifestações carenciais sensibilizam pouco os jovens ${ }^{4}$. O conhecimento sobre o que comer não instiga a mudança, mas funciona como um instrumento quando as pessoas desejam mudar ${ }^{1,3,4,10}$.

O contexto desafiador da educação nutricional exige o desenvolvimento de abordagens educativas que permitam abraçar os problemas alimentares em sua complexidade, tanto na dimensão biológica como na social e cultural ${ }^{4,9}$. É função do nutricionista ajudar as pessoas a modificarem seus hábitos e práticas alimentares, através da assistência nutricional a indivíduos e coletividade ${ }^{3,11,12}$. Para isso, a preparação básica do nutricionista com vista à prática pedagógica em nutrição tem lugar majoritariamente, por meio da disciplina de Educação Nutricional na graduação, que deve proporcionar ao futuro profissional a oportunidade de atuar ativamente em todos os níveis desse processo educativo $^{11}$.

Com o objetivo de desenvolver experiência na área de educação alimentar por parte de acadêmicos desta disciplina, foram propostas algumas atividades lúdicas e ilustrativas com alunos de uma escola pública. Embora tenha sido uma experiência de curta duração, buscou-se contribuir no conhecimento dos alimentos e na mudança de hábitos e práticas alimentares saudáveis dos escolares.

\section{DESCRIÇÃO DA EXPERIÊNCIA}

O trabalho constituiu-se de intervenção educativa com 17 escolares, de 10 a 12 anos, do sexto ano de uma escola Pública de Porto Alegre - RS. Este trabalho foi parte de uma atividade da disciplina de Educação Nutricional do curso de graduação em Nutrição, em que duas acadêmicas foram orientadas a realizar intervenções educativas semanais no período de 2 meses, totalizando 6 ações. Para tanto, foi utilizado o que preconiza Contento ${ }^{1}$, em que uma série de passos devem ser seguidos para a condução de um programa de educação alimentar e nutricional, que vão desde a análise das necessidades e do comportamento individual até o planejamento das ações/estratégias educativas. As ações educativas ocorreram em períodos disponibilizados pela escola, sempre com a presença da professora responsável pela turma e acompanhado pela professora acadêmica.

Depois do aprendizado teórico da disciplina desenvolveram-se atividades práticas no cenário escolar. O primeiro passo do planejamento foi identificar o grupo-alvo da intervenção na coletividade, levantar as necessidades recorrentes a uma determinada população e os potenciais problemas do grupo, sem esquecer-se de questões regionais, culturais, sociais, ambientais e psicológicas que estão muito relacionadas com a alimentação das pessoas. A partir desse dado que se formulou a metodologia utilizada ${ }^{1}$. 
O diagnóstico inicial do estado nutricional dos alunos foi feito pela Unidade Básica de Saúde responsável pela área de abrangência da escola. As aferições antropométricas foram realizadas na própria escola, conforme orientação da Organização Mundial de Saúde ${ }^{13}$. Para avaliação do estado nutricional, os alunos foram classificados pelo Índice de Massa Corporal (IMC) para a idade. O excesso de peso foi definido para alunos cujo IMC fosse maior ou igual $\mathrm{a}+1$ Z-escore, incluindo assim os adolescentes classificados como sobrepesos e obesos. Através desta avaliação antropométrica, detectou-se um alto percentual de excesso de peso $(33,4 \% \text { dos escolares })^{14}$. Esse dado foi repassado e assim prosseguiram-se as atividades. Os pais ou responsáveis assinaram um Termo de Autorização para utilização dos dados obtidos.

Ao iniciar a atividade na Escola, solicitou-se que os alunos preenchessem dois instrumentos de medida: a Escala de Preferência Alimentar ${ }^{15}$, que possui uma lista de frutas e vegetais e identifica se os alunos as conhecem ou não, e o quanto que os alunos gostam, variando de "detesto" para "adoro"; e o Formulário sobre Marcadores do Consumo Alimentar - SISVAN ${ }^{16}$, que pergunta em quantos dias da última semana o escolar consumiu determinados alimentos. A resposta varia de nenhum a todos os dias. Os alimentos mais "detestados" foram ervilha, chuchu, couve-flor, espinafre e vagem. E os mais "adorados": abacaxi, laranja e uva. Através dos Marcadores do Consumo Alimentar, verificou-se o baixo consumo de vegetais, já que, de 17 alunos, 11 responderam que não comeram salada crua (alface, tomate, pepino) na última semana ou comeram apenas em 1 dia da semana. O resultado foi semelhante para legumes e verduras cozidas (couve, abóbora, chuchu). Ao contrário das frutas, que foram consumidas todos os dias da semana por 9 alunos. Os outros dados ficaram controversos, visto que a variabilidade das respostas foi grande.

Também foi entregue aos pais ou responsáveis um questionário adaptado sobre o Comportamento Alimentar $^{17}$ dos seus filhos, entretanto, poucos retornaram. Este questionário avalia como os pais percebem o comportamento de seus filhos em relação a alimentação deles. Destes, as informações foram que a maioria dos alunos adora comida e se interessa pela mesma; terminam as refeições muito rapidamente; não deixam comida no prato ao final das refeições; não têm o hábito de tomar café da manhã e também não levam lanche de casa.

Ainda para o diagnóstico, planejou-se conhecer de outra maneira as Preferências Alimentares dos alunos para planejar as atividades seguintes. Para isso, simulou-se um Buffet de papel, onde cada aluno servia seu próprio prato de almoço com o que gostariam de comer. Foram disponibilizadas preparações mais saudáveis e outras nem tanto. Os alunos se serviam, colavam os alimentos em pratos de papel e pintavam conforme a cor do alimento. Observou-se a preferência por batata frita ao invés de batata assada (12 vs 5 alunos), refrigerante ao invés de suco (11 vs 6 alunos) e um consumo aleatório de laranja e/ou gelatina de sobremesa. A maioria dos alunos (15) se serviu de pelo menos uma hortaliça para compor seu prato.

O passo seguinte foi traçar os objetivos educacionais que pretendíamos alcançar. Estes descrevem como essa intervenção modificará os fatores preditivos que são os comportamentos de risco para determinadas doenças $^{1,18}$. Uma vez traçados o diagnóstico do comportamento alimentar dos alunos e os objetivos das ações, planejou-se estratégias lúdicas e participativas. O planejamento dessas ações foi pensado a partir da pesquisa na literatura, considerando as características dos indivíduos, como questões culturais, nível de escolaridade e habilidades físicas ${ }^{1}$ Também se considerou a duração da intervenção nutricional e as formas de avaliação, para que pequenas alterações nos hábitos alimentares fossem identificadas ${ }^{19}$.

$\mathrm{Na}$ primeira atividade foram proporcionados três vídeos breves, um sobre a propaganda/marketing infantil, outro sobre a quantidade de açúcar presente nos refrigerantes, e um terceiro sobre a transformação do açúcar em gordura. O objetivo do primeiro foi contribuir para o desenvolvimento crítico dos alunos com relação a propaganda de alimentos. Os dois vídeos seguintes tiveram como objetivo trabalhar o risco do consumo excessivo de açúcares e gorduras. Ilustrando de forma concreta, com um copo graduado, em conjunto com os alunos, se contou quantas colheres de sopa de 
açúcar há em uma latinha de refrigerante, e o quanto se consome durante uma semana. Para demonstrar a quantidade de gordura presente em um pacote de salgadinho e em uma colher de sopa de maionese industrializada, apresentou-se o equivalente com óleo vegetal. Utilizou-se um recipiente de vidro transparente para que os alunos visualizassem a quantidade de gordura que estaria sendo consumida, uma vez que a turma referiu utilizar quatro colheres de sopa de maionese quando consomem um hambúrguer.

$\mathrm{Na}$ sequência do trabalho, outra estratégia utilizada para transmitir conhecimento e esclarecer dúvidas foi um jogo de perguntas e respostas. A turma foi dividida em dois grupos e cada grupo respondeu a 12 perguntas. Toda vez que o grupo acertava a resposta, ganhava um ponto, como estímulo e reforço positivo. Após cada pergunta, realizou-se uma explanação sobre a resposta. Os alunos demonstraram bastante interesse e tinham muitas dúvidas durante a atividade.

Em outro encontro provaram-se diferentes frutas, sendo elas: kiwi, mamão, manga e tomate. Optou-se pelo kiwi por ser uma fruta não tão consumida e desconhecida por alguns alunos. A Escala aplicada no diagnóstico mostrou que muitos dos alunos não gostam de mamão e manga, por isso a escolha destas frutas. $E$ por fim, a escolha do tomate foi para salientar aos alunos que tomate é uma fruta e não uma verdura como muitos pensam. Todos os alunos, vendados, provaram e responderam a duas perguntas: "Que fruta é essa?" e "Você gostou?". O intuito principal foi estimular o consumo de frutas. A idéia era que os alunos provassem sem o preconceito (talvez já estabelecido) da parte visual. De uma forma geral, as frutas foram bem reconhecidas e bem aceitas pela maioria dos alunos. Com exceção do kiwi que foi a fruta menos conhecida.

Para o último encontro a turma foi para a sala de informática assistir um breve vídeo sobre o consumo de sal. O objetivo foi o mesmo da atividade realizada com açúcar e gordura. Em seguida, colocou-se uma mesa no centro da sala de aula com alimentos industrializados, sal de cozinha e alguns tipos de temperos. Foi demonstrada a quantidade de sal diária recomendada pela Organização Mundial de Saúde e esclareceu-se a diferença de sal e sódio. Ensinou-se como se analisa os rótulos dos alimentos e, com ajuda dos alunos, se calculou quantas gramas de sal há no macarrão, sopas instantâneas, salsicha, embutidos em geral e temperos prontos $^{20}$.

\section{DISCUSSÃo}

Ainda que se saiba sobre a necessidade de modificação dos hábitos e práticas alimentares da população, observa-se, na realidade brasileira, uma escassez de investigações dos possíveis fatores que interferem no desenvolvimento do comportamento alimentar $^{2}$. A maioria dos programas de educação nutricional e pesquisas publicadas nesta área não citam uma teoria ou um modelo particular que fundamente a prática ou a pesquisa ${ }^{3}$. Por isso o propósito de detalhar as estratégias utilizadas é contribuir para outras oportunidades de educação alimentar.

Com apenas seis encontros não foi possível perceber uma mudança no comportamento alimentar dos adolescentes. Segundo Rodrigues e $\mathrm{Boog}^{21}$, "as mudanças de comportamento são realizadas quando o adolescente percebe o sentido dessas em sua história de vida, que engloba o individual e o social, emoção e ação, compreensão dos fatos e segurança para manifestação e enfrentamento dos problemas." Por isso, deve-se investir em educação alimentar e nutricional em longo prazo, sendo necessárias estratégias adequadas aos sujeitos. No entanto, uma revisão sistemática com 13 estudos mostra que todos os trabalhos reportaram alguma alteração positiva após as intervenções, mesmo que não estatisticamente significativa, demonstrando a potencialidade destes programas para a promoção de uma vida mais saudável, independente do período de intervenção ${ }^{22}$.

Neste trabalho, ficou evidente a importância de serem utilizados materiais lúdicos para as ações educativas, bem como vídeos breves e atrativos. Oliveira et al. ${ }^{23}$, em um estudo com 70 escolares, concluíram que os alunos apresentaram maior conhecimento sobre alimentação e nutrição após o desenvolvimento da atividade educativa lúdica; esta intervenção é prazerosa e inovadora, podendo interferir e colaborar na escolha dos alimentos. 
Segundo o Ministério da Saúde e da Educação, o conhecimento sobre nutrição, por todos os profissionais que atuam no campo da alimentação e da educação, parece ser essencial para a melhora do quadro do estado nutricional das crianças brasileiras, pois estes contribuem na formação do comportamento alimentar ${ }^{24}$. Por isso, a relevância da presença dos acadêmicos de nutrição em sala de aula para a qualificação dos professores, já que o bacharelado de nutrição não permite a atuação do nutricionista em sala de aula.

O desenvolvimento deste trabalho permitiu constatar que estudantes de Nutrição da UFRGS necessitam de um maior conhecimento prático na área de psicologia e teorias da educação para lidar com os adolescentes. Esta atividade possibilita aprimoramento das competências e habilidades de comunicação, desenvolvimento de métodos e técnicas de ensino e formulação e execução de programas de educação nutricional. Sendo assim, a aplicação das atividades foi de suma importância para uma formação mais capacitada de futuros nutricionistas. A experiência adquirida prepara os graduandos a solucionarem imprevistos e a lidarem com ações educativas de acordo com as necessidades do público alvo.

As intervenções realizadas com os alunos podem ou não terem sido eficazes no curto prazo, contudo, uma base de conhecimento foi construída, proporcionando aos escolares condições para que possam refletir e tomar decisões mediante fatos percebidos.

\section{REFERÊNCIAS}

1. Contento IR. Nutrition Education: Linking Research, Theory, and Practice. Asia Pac J Clin Nutr. [periódico na internet]. 2008 [capturado 2013 Mar 20];17(1):176-9. Disponível em: http://apjcn.nhri.org.tw/server/apjcn/ volume17/vol17suppl.1/176-179S10-1.pdf

2. Ramos M, Stein LM. Desenvolvimento do comportamento alimentar infantil. J Pediatria. 2000;76(Supl.3):S229-37.

3. Assis MAA, Nahas MV. Aspectos motivacionais em programas de mudança de comportamento alimentar. Rev Nutr. [periódico online] 1999 [capturado 2013 Mar 20];12(1):33-41. Disponível em: http://www.scielo.br/pdf/rn/ v12n1/v12n1a03.pdf.

4. Boog MCF, Vieira CM, Oliveira NL, Fonseca O, L'abbate S. Utilização de vídeo como estratégia de educação nutricional para adolescentes: "comer... o fruto ou o produto?" Rev Nutr. [periódico online] 2003 [capturado 2013 Mar 20];16(3):281-93. Disponível em: http://www.scielo.br/scielo.php?script=sci_arttext\&pid=S14 15-52732003000300006
5. Brug J. Determinants of healthy eating: motivation, abilities and environmental opportunities. Family Pract Adv. [periódico online] 2008 [capturado 2013 Mar 20];25(suppl 1):i50-i5. Disponível em: http://fampra.oxfordjournals.org/ content/25/suppl_1/i50.abstract

6. Rossi A, Moreira EAM, Rauren MS. Determinantes do comportamento alimentar: uma revisão com enfoque na família. Rev Nutr. 2008;21(6):739-48.

7. Sousa AA, Bueno MP, de Araújo GC, Filho DOL. Fatores motivacionais determinantes na compra de produtos alimentícios. In: XIII Simpósio de Engenharia de Produção (SIMPEP), Bauru, SP, Brasil, 06 a 08 nov 2006. Bauru (SP): Departamento de Engenharia de Produção da Universidade Estadual Paulista; 2006.

8. Instituto Brasileiro de Geografia e Estatística. POF 20082009: desnutrição cai e peso das crianças brasileiras ultrapassa padrão internacional. Brasília (DF): IBGE; 2010.

9. Boog MCF. Educação nutricional: Passado, presente, futuro. Rev Nutr PUCCAMP [periódico online]. 1997 [capturado 2013 Mar 20];10(1):5-19. Disponível em: http://www.faculdadeguararapes.edu.br/site/hotsites/bibliot eca/educacaonutricional_passado-presentefuturo59500.pdf.

10. Mendonça DRB. A Importância da Educação Nutricional. Nutrição e Ciência, Sociedade Brasileira de Diabetes, 2006. [capturado 2013 Mar 20]. Disponível em: http://www.diabetes.org.br/alimentos/181-a-importanciada-educacao-nutricional.

11. Bizzo MLG, Leder L. Educação nutricional nos parâmetros curriculares nacionais para o ensino fundamental. Rev Nutr. [periódico online].2005 [capturado 2013 Mar 20];18(5). Disponível em: http://dx.doi.org/10.1590/S141552732005000500009 .

12. Brasil. Ministério da Educação. Conselho Nacional de Educação. Diretriz Curricular da Nutrição. Parecer CNE/CES no 1.133, de 7 de agosto de 2001. Brasília (DF): Ministério da Educação; 2001.

13. World Health Organization. Physical Status: the use and interpretation of anthropometry. Geneva: WHO; 1995. (WHO Technical Report Series; no 854).

14. Wagner DGP. Prevalência de excesso de peso em crianças e adolescentes cadastrados no SISVAN-WEB pertencentes à região de abrangência de uma unidade básica de saúde de Porto Alegre [trabalho de conclusão de curso]. Porto Alegre (RS): Universidade Federal do Rio Grande do Sul; 2012.

15. Ramos M. Influência das estratégias e preferências maternas no comportamento alimentar infantil [mestrado]. Porto Alegre (RS): Pontifícia Universidade Católica do Rio Grande do Sul; 2001.

16. Brasil. Ministério da Saúde. Secretaria de Atenção à Saúde. Departamento de Atenção Básica. Protocolos do Sistema de Vigilância Alimentar e Nutricional - SISVAN na assistência à saúde. Brasília (DF): Ministério da Saúde; 2008.

17. Viana V, Sinde S. O comportamento alimentar em crianças: estudo de validação de um questionário numa amostra portuguesa (CEBQ). Anál Psicol. [periódico online] 2008 [capturado 2013 Mar 20];1(XXVI):111-20. Disponível em: http://www.scielo.oces.mctes.pt/pdf/aps/ v26n1/v26n1a09.pdf

18. Lytle LA, Perry CL. Applying research and theory in program planning: an example from a nutrition education intervention. Health Promot Pract. 2001;2(1):68-80.

19. Toral N, Slater B. Intervention Based Exclusively on Stage-Matched Printed Educational Materials Regarding Healthy Eating Does Not Result in Changes to 
Adolescents' Dietary Behavior. ScientificWorldJournal. [periódico online] 2012 [capturado 2013 Mar 20]. 2012:174640. doi:10.1100/2012/174640. [Disponível em: http://www.hindawi.com/journals/tswj/2012/174640/.

20. Agência Nacional de Vigilância Sanitária. Rotulagem nutricional obrigatória: manual de orientação aos consumidores - Educação para o consumo saudável. Alimentos. Brasília (DF): Ministério da Saúde, ANVISA; 2008. 17p.

21. Rodrigues EM, Boog MCF. Problematização como estratégia de educação nutricional com adolescentes obesos. Cad Saúde Pública 2006;22(5):923-31.

22. Souza EA, Filho VCB, Nogueira JAD, Júnior MRA. Atividade física e alimentação saudável em escolares brasileiros: revisão de programas de intervenção. Cad Saúde Pública 2011;27(8):1459-71.

23. Oliveira JC, Costa SD, Da Rocha SMB Educação nutricional com atividade lúdica para escolares da rede municipal de ensino de Curitiba. Cad Escola de Saúde 2011;2(6):100-6.

24. Brasil. Ministério da Saúde. Secretaria-Executiva. Departamento de Apoio à Gestão Descentralizada. Colegiado de gestão regional na região de saúde intraestadual: orientações para organização e funcionamento. Brasília (DF): Ministério da Saúde; 2009. (Série B.Textos Básicos em Saúde); (Série Pactos pela Saúde 2006, v. 10).

\section{Endereço para correspondência:}

Maurem Ramos

Rua Ramiro Barcelos $n^{\circ} 2400$

Porto Alegre/RS - CEP 90035-903

Telefone: +55 5133085238

E-mail: maurem.profnut@gmail.com 